\title{
Depression and elevation tsunami waves in the framework of the Korteweg-de Vries equation
}

\author{
R. Grimshaw ${ }^{1}$ C. Yuan ${ }^{1}$
}

Received: 15 January 2016/Accepted: 9 July 2016/Published online: 14 September 2016

(C) The Author(s) 2016. This article is published with open access at Springerlink.com

\begin{abstract}
Although tsunamis in the deep ocean are very long waves of quite small amplitudes, as they propagate shorewards into shallow water, nonlinearity becomes important and the structure of the leading waves depends on the polarity of the incident wave from the deep ocean. In this paper, we use a variable-coefficient Korteweg-de Vries equation to examine this issue, for an initial wave which is either elevation, or depression, or a combination of each. We show that the leading waves can be described by a reduction of the Whitham modulation theory to a solitary wave train. We find that for an initial elevation, the leading waves are elevation solitary waves with an amplitude which varies inversely with the depth, with a pre-factor which is twice the maximum amplitude in the initial wave. By contrast, for an initial depression, the leading wave is a depression rarefaction wave, followed by a solitary wave train whose maximum amplitude of the leading wave is determined by the square root of the mass in the initial wave.
\end{abstract}

Keywords Tsunami $\cdot$ Solitary wave $\cdot$ Shoaling

\section{Introduction}

The study of tsunamis can be described as four phases. First, there is the generation usually by a submarine earthquake, although submarine landslides and volcanic eruptions have also been known to produce devastating tsunamis. Second, in the deep ocean a tsunami is essentially a long water wave of relatively small amplitude and hence propagates without significant change of form at a speed $\sqrt{g h}$ where $h$ is the ocean depth. In this phase, the tsunami can be either a wave of depression, or a wave of elevation, or a combination of these, see the recent assessments by Didenkulova et al. (2007), Arcas and Segur (2012) and

R. Grimshaw

r.grimshaw@ucl.ac.uk

1 Department of Mathematics, University College London, London, UK 
Dias et al. (2014). In the third phase, the tsunami wave propagates shorewards from the deep ocean over the continental slope and shelf into shallow water. In this process, the wavelength decreases and the wave amplitude increases, indicating that both wave dispersion and nonlinearity become important. Finally in the fourth phase, the tsunami impacts the coast, and here, turbulence and the potential to carry debris become important factors.

In this paper, we are concerned with the third phase, where the increasing effect of nonlinearity may lead to quite different outcomes, depending on the wave polarity as the wave transverses the deep ocean, see Carrier et al. (2003) and Fernando et al. (2008) for instance. Although initial depression waves are potentially just as damaging as initial elevation waves, they have not received the same attention, although we note the theoretical studies by Tadepalli and Synolakis (1994, 1996), and most recently by Grimshaw et al. (2015), the analysis of field data by Soloviev and Mazova (1994), and the experiments of Kobayashi and Lawrence (2004), Klettner et al. (2012), Rossetto et al. (2011) and Charvet et al. (2013).

Many studies of the development of a tsunami as it approaches the shore have used the nonlinear shallow water equations to examine the connection between the incident wave mass, amplitude and polarity, on the shoreline impact, see for instance Tadepalli and Synolakis (1994, 1996), Carrier et al. (2003), Madsen and Schaffer (2010) and Didenkulova and Pelinovsky (2011). In particular, studies by Didenkulova (2009), Didenkulova et al. (2006, 2007) and Pelinovsky (2006) using the nonlinear shallow water equations have elucidated the role of initial steepness in increasing the eventual run-up height, and we especially note that Didenkulova et al. (2014) found that this nonlinear steepness effect was enhanced when the initial wave was one of depression.

However, although the nonlinear shallow water equations have been widely used and have yielded valuable and insightful results, they are non-dispersive and hence do not capture the effects of wavenumber dispersion which generically develops as the tsunami propagates shorewards generating shorter length scales. In particular, the shocks which can arise in nonlinear shallow water equations need to be resolved, either with a turbulent wave-breaking scheme or with wave dispersion. As in the recent work by Grimshaw et al. (2015), in this paper we make the latter choice and invoke a Korteweg-de Vries (KdV) model to examine the contrasting evolution of elevation and depression waves as they propagate into a region of decreasing depth.

Indeed, it is well known that the combination of weak nonlinearity and weak linear dispersion leads to a $\mathrm{KdV}$ equation, or more generally to a Boussinesq system, see Segur (2007) and related articles in the book by Kundu (2007) for a tsunami context. However, most studies of tsunamis using the $\mathrm{KdV}$ equation have focussed on the classical solitary wave solution, which is always an elevation wave. Hence, in this paper, we use a variablecoefficient $\mathrm{KdV}$ equation to examine the evolution of depression waves vis-a-vis the evolution of elevation waves and further the evolution of an "up-down" wave, that is an elevation followed by a depression, and also a "down-up" wave, that is a depression followed by an elevation. In particular, this will emphasise the important role of the undular bore solutions of the $\mathrm{KdV}$ equation, as seen in some tsunami observations and numerical simulations, see Arcas and Segur (2012) and Grue et al. (2008) for instance. It is pertinent to note here that the critique of the validity of $\mathrm{KdV}$ models by Madsen et al. (2008), Madsen and Schaffer (2010) and Arcas and Segur (2012), amongst others, is based on solitary wave dynamics, and we suggest that this is an overly restrictive view of the value of $\mathrm{KdV}$ models. 
In Sect. 2, we present the KdV Eq. (1) (see below) for water waves on a variable depth. Our aim is to describe the evolution of an elevation wave and contrast that with the evolution of a depression wave. For this purpose, we use the Whitham modulation equations for a modulated periodic wave train in variable depth, and these are presented in Sect. 3. Because our main interest is in the amplitude of the leading wave in the evolving wave train, the full Whitham modulation equations are reduced to the simpler equations for a solitary wave train. Then, in Sect. 4, we describe the long-time outcome of the initial value problem and find asymptotic descriptions for the leading wave amplitude in both the elevation and depression cases. These results are known for waves on a constant depth, and here, we show how those results can be extended to variable depth. We also consider the outcome from an initial "up-down" wave and an initial "down-up" wave. Readers wishing to avoid the detailed analysis can go to the discussion in Sect. 5 and the plots of our simulations.

\section{Korteweg-de Vries equation}

The KdV equation for water waves over variable depth was derived by Johnson (1973a, 1973b), see also the review by Grimshaw (2007b). A similar general equation for internal waves was derived by Grimshaw (1981), see also the reviews by Grimshaw (2007a), Grimshaw et al. (2010), which includes surface water waves as a special case. When expressed in terms of the usual physical variables for the free surface elevation $\zeta(x, t)$ on a variable depth $h(x)$, it is

$$
\zeta_{t}+c \zeta_{x}+\frac{c_{x}}{2} \zeta+\frac{3 c}{2 h} \zeta_{x}+\frac{c h^{2}}{6} \zeta_{x x x}=0, \quad c=\sqrt{h}
$$

This is based on non-dimensional variables using a timescale $\sqrt{h_{0} / g}$ and a length scale $h_{0}$ where $h_{0}$ is a measure of the water depth. The first two terms in (1) are the dominant terms and, by themselves, describe the propagation of a linear long wave with speed $c(x)=\sqrt{h(x)}$. For tsunami waves, we cast (1) into an asymptotically equivalent form describing evolution along the wave path determined by the linear wave speed, see for instance Grimshaw et al. (2015),

$$
\zeta_{\tau}+\frac{c_{\tau}}{2 c} \zeta+\frac{3}{2 h} \zeta \zeta_{X}+\frac{h}{6} \zeta_{X X X}=0
$$

where

$$
\tau=\int_{0}^{x} \frac{\mathrm{d} x^{\prime}}{c\left(x^{\prime}\right)}, \quad X=\tau-t, \quad c=h^{1 / 2}
$$

Here $\tau$ is a time-like variable measuring travel time along the wave path, and now $h=h(\tau)$.

It is now convenient to cast (2) can into an exactly equivalent form. First,

$$
U=\frac{3 c^{1 / 2} \zeta}{2}, \quad U_{\tau}+\frac{1}{h c^{1 / 2}} U U_{X}+\frac{h}{6} U_{X X X}=0
$$

and then make a further transformation to get

$$
U_{T}+6 U U_{X}+\beta(T) U_{X X X}=0,
$$


where

$$
T=\frac{1}{6} \int_{0}^{\tau} \frac{\mathrm{d} \tau^{\prime}}{h\left(\tau^{\prime}\right) c^{1 / 2}\left(\tau^{\prime}\right)}=\frac{1}{6} \int_{0}^{x} \frac{\mathrm{d} x^{\prime}}{h\left(x^{\prime}\right)^{7 / 4}}, \quad \beta=h^{9 / 4} .
$$

On a constant depth $h=1, c=1$ and $\beta=1$, while on a variable depth as $h$ decreases (increases), $c, \beta$ also decrease (increase). There are two important conservation laws,

$$
\begin{gathered}
U_{T}+\left\{3 U^{2}+\beta U_{X X}\right\}_{X}=0 \\
\left\{\frac{U^{2}}{2}\right\}_{T}+\left\{2 U^{3}+\beta U U_{X X}-\beta \frac{U_{X}^{2}}{2}\right\}_{X}=0 .
\end{gathered}
$$

corresponding to conservation of mass and wave action flux, respectively.

On a constant depth when the coefficient $\beta$ is a constant, the $\mathrm{KdV}$ equation (5) has the well-known solitary wave (soliton) solution, relative to a pedestal $d$,

$$
U=a \operatorname{sech}^{2}(\Gamma(X-V T))+d, \quad V-6 d=2 a=4 \beta \Gamma^{2} .
$$

More generally, when the coefficient $\beta$ is a constant, the $\mathrm{KdV}$ equation (5) supports a periodic travelling wave $U(X-V T)$, the well-known cnoidal wave solution

$$
U=a\left\{b(m)+\mathrm{cn}^{2}(\gamma \theta ; m)\right\}+d, \quad \theta=k(X-V T),
$$

where

$$
\begin{gathered}
a=2 m \beta \gamma^{2} k^{2}, \quad b(m)=\frac{1-m}{m}-\frac{E(m)}{m K(m)}, \\
V-6 d=2 a\left\{\frac{2-m}{m}-\frac{3 E(m)}{m K(m)}\right\}=4 \beta \gamma^{2} k^{2}\left\{2-m-\frac{3 E(m)}{K(m)}\right\} .
\end{gathered}
$$

Here $\operatorname{cn}(x ; m)$ is the Jacobian elliptic function of modulus $m, 0<m<1$, and $K(m)$ and $E(m)$ are the elliptic integrals of the first and second kind. The expression (10) has period $2 \pi$ in $\theta$ so that $\gamma=K(m) / \pi$, while the spatial period is $2 \pi / k$. The (trough-to-crest) amplitude is $a$, and the mean value over one period is $d$. It is a three-parameter family with parameters $k, m, d$ say. As the modulus $m \rightarrow 1$, this becomes a solitary wave, since then $b \rightarrow 0$ and $\operatorname{cn}(x) \rightarrow \operatorname{sech}(x)$, while $\gamma \rightarrow \infty, k \rightarrow 0$ with $\gamma k=\Gamma$ fixed. As $m \rightarrow 0, b \rightarrow-1 / 2, \gamma \rightarrow 1 / 2, \operatorname{cn}(x) \rightarrow \cos (x)$, and it reduces to a sinusoidal wave $(a / 2) \cos (\theta)$ of small amplitude $a \sim m$ and wavenumber $k$.

\section{Modulation equations}

The Whitham modulation theory allows this cnoidal wave (10) to vary slowly with $T, X$, that is the wave parameters such as wavenumber $k$, modulus $m$ and mean level $d$ vary slowly with $T, X$. The Whitham modulation equations for a constant-coefficient $\mathrm{KdV}$ equation can be obtained by averaging conservation laws, the original Whitham method, see Whitham $(1965,1974)$, or by exploiting integrability, see Kamchatnov (2000) for instance. But here we are concerned with the case when $\beta=\beta(T)$ varies slowly with $T$, and since the variable-coefficient $\mathrm{KdV}$ equation (5) is not integrable, we will use the original Whitham method, readily adapted to this situation, see Grimshaw (2007a) for 
instance. A similar strategy was used by Myint and Grimshaw (1995) for a frictionally perturbed KdV equation. An alternative method developed by Kamchatnov (2004) for a perturbed $\mathrm{KdV}$ equation requires a change of variable in (5), $U=\beta \tilde{U}$ and $\tilde{T}=\int^{\mathrm{T}} \beta \mathrm{d} T$, to generate a $\mathrm{KdV}$ equation for $\tilde{U}$ with a perturbation term of the form $\beta_{\tilde{T}} \tilde{U} / \beta$. This approach was used by El et al. $(2007,2012)$ to study the evolution of solitary waves and undular bores over a slope, a study similar but complementary to that described here.

As three modulation equations are needed, we supplement $(7,8)$ with the equation for conservation of waves,

$$
k_{T}+(k V)_{X}=0 .
$$

The remaining two modulation equations are obtained by inserting the cnoidal wave solution into the conservation laws $(7,8)$ and averaging over the phase $\theta$. The outcomes are

$$
\begin{gathered}
d_{T}+6 P_{X}=0, \quad P=\left\langle\frac{U^{2}}{2}\right\rangle, \\
P_{T}+Q_{X}=0, \quad Q=\left\langle 2 U^{3}-\frac{3 \beta U_{X}^{2}}{2}\right\rangle,
\end{gathered}
$$

where the $\langle\cdots\rangle$ denotes a $2 \pi$-average over $\theta$. The expressions $P, Q$ are given by, see Grimshaw (2007a, b),

$$
\begin{gathered}
P=\frac{d^{2}}{2}+\frac{a^{2}}{2}\left\{C_{4}-b^{2}\right\}, \\
C_{4}=\frac{1}{3 m^{2} K(m)}\left\{3 m^{2} K(m)-5 m K(m)+4 m E(m)+2 K(m)-2 E(m)\right\} \\
Q=\left\{-4 d^{3}+12 d P+6 a^{3}\left\{-\frac{2 b^{3}}{3}+\frac{(1-m) b}{2 m}+\left(b+\frac{1-2 m}{2 m}\right) C_{4}+\frac{5}{6} C_{6}\right\}\right\}, \\
C_{6}=\frac{1}{15 m^{3} K(m)}\left\{15 m^{3} K(m)-34 m^{2} K(m)+23 m^{2} E(m)+27 m K(m)\right. \\
-23 m E(m)-8 K(m)+8 E(m)\} .
\end{gathered}
$$

Here the notations $C_{4}, C_{6}$ denote the average values of $\mathrm{cn}^{4}$ and $\mathrm{cn}^{6}$, respectively, over one period and depend on the modulus $m$ only.

As our main concern is with the front of the developing wave trains, where typically the modulus $m \approx 1$, and the wave train can be described as a solitary wave train. Hence, we take the limit $m \rightarrow 1$ when $b \sim-1 / K(m), C_{4} \sim 2 / 3 K(m)$, and $C_{6} \sim 8 / 15 K(m)$. To leading order $P \sim d^{2} / 2$ and $Q \sim d^{3} / 3$ and then both equations $(14,15)$ reduce to the same equation for $d$ alone,

$$
d_{T}+6 d d_{X}=0,
$$

and so $d$ can be regarded as a known quantity. The cnoidal wave expression (10) now becomes a solitary wave train riding on a pedestal,

$$
\left.U=a \operatorname{sech}^{2}(\Gamma(X-V T) ; m)\right\}+d, \quad V-6 d=2 a=4 \beta \Gamma^{2},
$$

with one parameter to be determined. This is provided by a reduced form of the wave action equation, 


$$
\left\{\frac{a^{2}}{k \gamma}\right\}_{\tau}+V\left\{\frac{a^{2}}{k \gamma}\right\}_{X}+\frac{a^{2}}{k \gamma} 6 d_{X}=0,
$$

This can be obtained by a more careful consideration of the limit $m \rightarrow 1$ in the modulation equations $(14,15)$ by retaining the terms in $1 / K(m)$, or more directly by averaging the wave action conservation law (8) for a solitary wave, see Whitham (1974) for the case of the constant-coefficient KdV equation, or Grimshaw (1979) and the discussion in El et al. (2012) for the variable-coefficient KdV equation. The pair (13), (20) form a nonlinear hyperbolic system for a solitary wave train and can be solved explicitly. Indeed, using the expressions in (19), (20) can be written as

$$
\mathcal{A}_{T}+(6 d+2 a) \mathcal{A}_{X}+\mathcal{A} 6 d_{X}=0, \quad \mathcal{A}=\left(\beta a^{3}\right)^{1 / 2} .
$$

This is an equation for the amplitude $a$ alone and is readily solved using characteristics. Then, with $a, d$ and hence $V$ known, the wavenumber $k$, where $\Gamma=k \gamma$, can be found from (13) for the conservation of waves, which is then a linear hyperbolic equation for $k$.

\section{Evolution over a slope}

We shall suppose henceforth that the depth $h=1$ in the region $T<0$ and then decreases monotonically to a constant value $h_{1}<1$ as $T \rightarrow 2 T_{1}$ and remains constant thereafter. The scale for this variability is $T_{1}$ and it will be assumed that this is much greater than the intrinsic scale of the leading evolving solitary wave. Correspondingly, $\beta=1, T<0$, and then decreases monotonically to $\beta_{1}<1, T>2 T_{1}$. Note that the length of the slope in physical space is readily found by inverting (6)

$$
x_{s}=6 \int_{0}^{2 T_{1}} \beta^{7 / 9}(T) \mathrm{d} T .
$$

In this paper, we are concerned with the "initial" value problem when $U=U_{0}(X)$ at $T=0$, which is a specification of a wave at an initial location. Using the transformations $(3,4,6)$, this corresponds to a specification $\zeta=\zeta_{0}(t)=2 U_{0}(-t) / 3$ at $x=0$. The $\mathrm{KdV}$ equation (5) then describes the spatial evolution of $U(X, T)$. Further, we suppose that $U_{0}(X)$ has compact support and that $U_{0}(X)=0$ for $X>0$, so that at $T=0$ the initial wave is located where the depth is a constant. The aim is then to describe the evolution of the wave as it propagates into the region of decreasing depth, emphasising the key difference between an initial elevation, $U_{0}(X) \geq 0$ and an initial depression, $U_{0}(X) \leq 0$. Two key parameters are its maximum/minimum value $\pm U_{M}$, respectively, and the mass

$$
M=\int_{-\infty}^{\infty} U_{0}(X) \mathrm{d} X
$$

Note that from (7) $M$ is conserved by the $\mathrm{KdV}$ equation (5).

In both cases, we use an adaptation of the Gurevich-Pitaevskii asymptotic method based on the Whitham modulation equations, Gurevich and Pitaevskii (1974) and subsequently developed by many others, see El (2007) for a recent review. In this approach, we at first consider the initial value problem for the Hopf equation

$$
U_{T}+6 U U_{X}=0, \quad U(X, T=0)=U_{0}(X) .
$$


This is obtained from (5) by omitting the dispersive term $U_{X X X}$, and formally this requires that at least initially, dispersive effects are weak. That is, $U_{0}(X)$ varies rather slowly with $X$. Significantly note that in this asymptotic limit, there is no dependence on $\beta$. The Hopf equation can be readily solved using characteristics and the solution of (24) is given implicitly by

$$
U=U_{0}(X-6 U T) .
$$

This is valid up to a time $T_{0}$ (it is assumed that $T_{0}<2 T_{1}$ ) when a shock forms at the place $X_{0}$, and after that time the shock must be resolved by the reintroduction of the dispersive term in the full $\mathrm{KdV}$ equation. In the Gurevich-Pitaevskii asymptotic method, this is achieved using the Whitham modulation equations, so that the shock is resolved by a modulated periodic wave train, sometimes referred to as a dispersive shock wave or undular bore. Here, we note that $T_{0}, X_{0}$ are given by

$$
T_{0}=\frac{1}{-6 U_{X}\left(X_{m}\right)}, \quad U_{X}\left(X_{m}\right)=\min \left(-U_{X}\right), \quad X_{0}=X_{m}+U_{0}\left(X_{m}\right) T_{0} .
$$

As is well known, $X_{m}$ is the location of the maximum negative slope in the initial profile. In the elevation case, the shock forms on the front face of the initial profile and in the depression case it forms on the rear face.

In the following subsections, we use this method to analyse the evolution of the leading waves from an initial elevation, an initial depression and from a "up-down" and "downup" initial condition. The analysis is supported by numerical simulations of (5) where

$$
\beta(T)=\frac{\left(1+\beta_{1}\right)}{2}-\frac{\left(1-\beta_{1}\right)}{2} \tanh \left(\kappa\left(T-T_{1}\right)\right),
$$

where $\kappa$ is chosen so that $\kappa T_{1} \gg 1$ and then $\beta(T)$ varies smoothly and slowly from 1 when $T<0$ to $\beta_{1}<1$ when $T>2 T_{1}$. Correspondingly, the depth $h$ varies from 1 at $x=0$ to $h_{1}=\beta_{1}^{4 / 9}$ at $x=x_{s}$ (22). In our simulations, we set $\beta_{1}=0.333$ and $T_{1}=4$ so that $h_{1}=$ 0.614 and $x_{s}=34.5$.

The initial condition $U_{0}(X)$ for the numerical simulations is specified by a box-like profile for the cases of an initial elevation or depression,

$$
U_{0}(X)=\frac{U_{M}\left\{\tanh \left(\Gamma_{0}(X+3 L)\right)-\tanh \left(\Gamma_{0}(X+L)\right)\right\}}{2 \tanh \left(\Gamma_{0} L\right)}, \quad M=\frac{2 U_{M} L}{\tanh \left(\Gamma_{0} L\right)} .
$$

This has a maximum (minimum) value of $U_{M}$ at $X=-2 L$ where $L>0$ is chosen so that $\Gamma_{0} L \gg 1$, and then $U_{0}(X)$ is approximately zero in $X>0$ as required. It is effectively a box of height $U_{M}$, length $2 L$ and mass $M=2 U_{M} L$. Note that then $X_{m}=-L,-3 L$ according as the initial condition is one of elevation or depression, and that from (26) $T_{0}= \pm 1 / 3 U_{M} \Gamma_{0}, X_{0}=-L+1 / 6 \Gamma_{0},-3 L-1 / 6 \Gamma_{0}$. If the leading solitary wave has an amplitude $a$ and a timescale of $1 / \gamma k V$, see (19), we require that the variation of $\beta$ with $T$ be much slower than this, that is, $1 / \gamma k V \ll T_{1}$. Using the estimates of $a$ obtained below, we choose $T_{1}$ accordingly. Several simulations were performed, and a typical set of parameters are $U_{M}= \pm 1, \Gamma_{0}=0.5, L=16, \beta_{1}=0.333, T_{1}=4$ and $\kappa=0.75$; these lead to the corresponding values $M= \pm 32, T_{0}=0.667, X_{0}=-0.667,-3.333$ and $\beta_{0}=\beta\left(T_{0}\right)=0.99$. For an "up-down" or "down-up" wave, the initial boxes (28) are combined into 


$$
\begin{aligned}
U_{0}(X)= & \frac{U_{M}\left\{\tanh \left(\Gamma_{0}(X+3 L)\right)-\tanh \left(\Gamma_{0}(X+L)\right)\right\}}{2 \tanh \left(\Gamma_{0} L\right)} \\
& -\frac{U_{M}\left\{\tanh \left(\Gamma_{0}(X+7 L)\right)-\tanh \left(\Gamma_{0}(X+5 L)\right)\right\}}{2 \tanh \left(\Gamma_{0} L\right)},
\end{aligned}
$$

with similar parameter settings. The numerical simulations of the KdV equation (4) were performed using a pseudo-spectral code, based on a Fourier interpolant.

\subsection{Initial elevation}

It is well known that for the constant-coefficient $\mathrm{KdV}$ equation, the initial wave will evolve into a solitary wave train with a finite number $N$ of rank-ordered solitary elevation waves, together will some small-amplitude trailing and dispersing radiation. This can be established using the inverse scattering transform, see Ablowitz and Segur (1981) or Drazin and Johnson (1989), or when $N$ is large, using the Whitham modulation equations, see El (2007) for instance.

Here, when the depth varies, we adapt and simplify the Gurevich-Pitaevskii approach, guided here by the detailed theory developed by El et al. (2012) for the propagation of an undular bore up a slope. The main difference is that in El et al. (2012) the undular bore was formed from a step initial condition (actually a box but much longer than (28) that we have used here). Consequently, the undular bore in El et al. (2012) was well formed when entering the slope, and in particular there was no sign in their simulations of the rarefaction forming at the rear of the evolving disturbance, see our simulations below. Thus, as described above by (25), the solution initially develops according to the Hopf equation and a shock forms at a time $T_{0}$ and place $X_{0}$ (26). This is resolved here by an undular bore, whose leading solitary waves are riding on an undisturbed level, since the solution is zero ahead of the shock. Thus, we set $d=0$ in (18) and seek a similarity solution of (21) for large $N$ and large $T$. This is given by

$$
\beta^{1 / 3} a \sim \frac{X-X_{0}}{2 \sigma}, \quad 0<\frac{X-X_{0}}{2 \sigma}<a_{0} \quad \sigma=\int_{T_{0}}^{\mathrm{T}} \frac{\mathrm{d} T^{\prime}}{\beta^{1 / 3}\left(T^{\prime}\right)} .
$$

Here $a_{0}$ is a constant and the leading solitary wave obeys the adiabatic law $a \sim a_{0} / \beta^{1 / 3}$ which in terms of the original variables $\zeta, h$ is the well-known result that the amplitude for $\zeta$ varies as $h^{-1}$. In turn the constant $a_{0}$ can be found using the theory of Gurevich and Pitaevskii (1974) for a constant depth, see El (2007), or the adaptation of that theory for a slope by El et al. (2012). Since the shock solution of the Hopf equation develops a jump of $U_{M}$, the outcome is that $a_{0}=2 U_{M} \beta_{0}$ where $\beta_{0}=\beta\left(T_{0}\right)$. Note that this asymptotic solution describes only the leading solitary wave train, and as shown by El et al. (2012) it will in general be followed by a more complex nonlinear dispersive wave train. Here, we do not attempt to match with the rest of the bore, and are content with using just this long-time similarity solution. This does not require the matching used in El et al. (2012), who essentially solved (21) (with $d=0$ ) using characteristics with "initial" conditions at the front of the undular bore. Here, we are concerned mainly with the leading amplitude given by (29). The corresponding general solution of (13) for the wavenumber is

$$
k=\frac{1}{\sigma} k_{0}\left(\frac{X-X_{0}}{2 \sigma}\right)
$$


where $k_{0}(\cdot)$ is an arbitrary function, determined by matching the solution with the trailing wave train, a task which is beyond the scope of this article.

A typical simulation is shown in Fig. 1, where $\beta$ varies from 1 (top panel) to $2 / 3$ (middle panel) to $1 / 3$ (bottom panel), which corresponds to $h=1,0.835,0.614$, respectively. Each plot is at a fixed $T$, that is at a fixed place $x$ and hence at a fixed depth $h$, while as $X$ varies;

Fig. 1 Time series from the simulation with the initial condition (28) with $U_{M}=$ $1, \Gamma_{0}=0.5, L=16$ and $\beta_{1}=0.333, T_{1}=4, \kappa=0.75$. $T=0, \beta=1, h=1$, top panel; $T=T_{1}, \beta=-0.667, h=0.835$, middle panel;

$T=2 T_{1}, \beta=0.333, h=0.614$, bottom panel
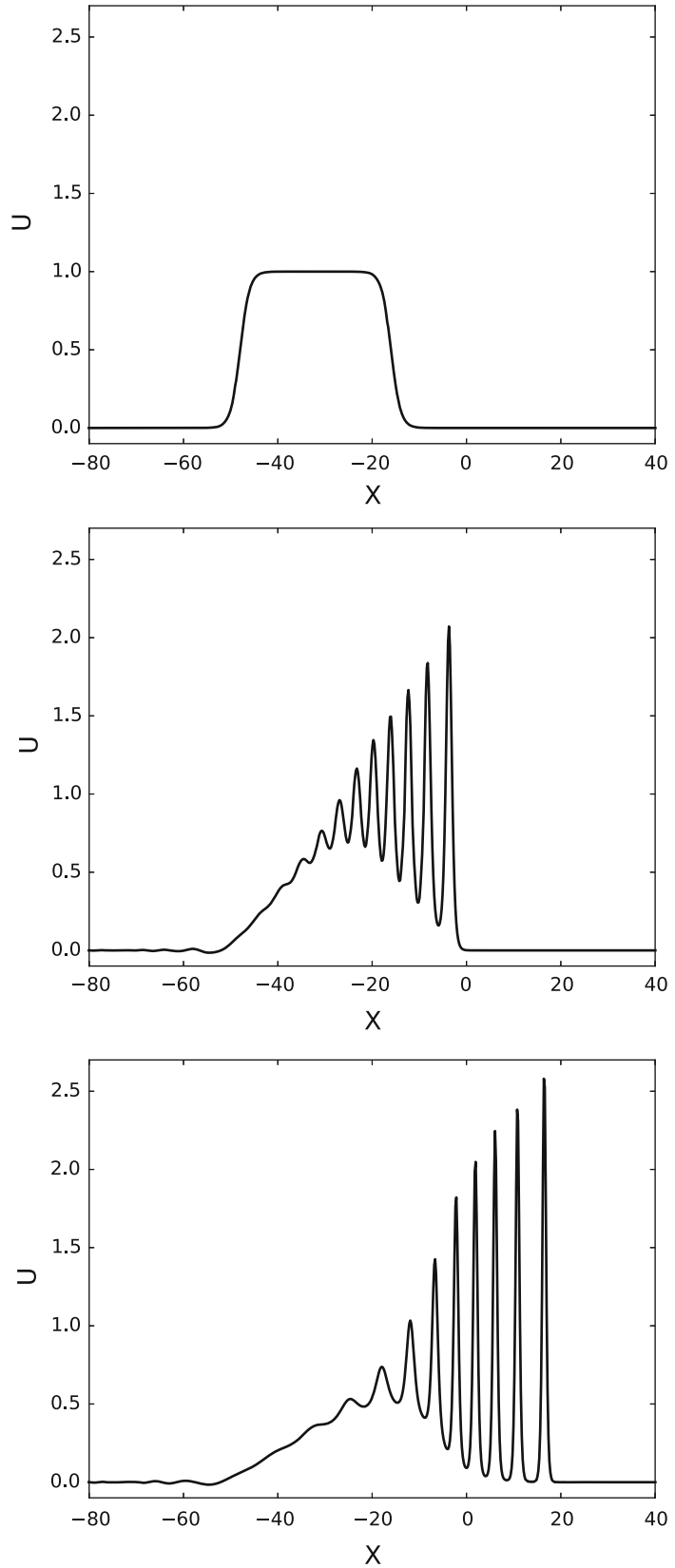
with $x$ fixed, these are then time series in $t$, the physical (non-dimensional) time. Apart from the factor $3 c^{1 / 2} / 2$ in the transformation between the physical wave height $\zeta$ and $U$ in (4), these are what would be observed at each corresponding depth. Note that this factor varies as 1.5, 1.434, 1.328 and is not a hugely significant feature. It follows that the nonlinear dynamics seen here in the transformed equation is completely replicated in the original physical variables. Also note that the length of the shoaling region $2 T_{1}=8$ in the transformed equation transforms to the length $x_{s}=34.5$. There is very good qualitative agreement with this asymptotic theory. Quantitatively, the leading solitary wave has a predicted amplitude at $T=2 T_{1}$ of $2 U_{M} \beta_{0} / \beta_{1}^{1 / 3}=2.88$ which is greater than the value of approximately 2.5 in the simulation. This is because in the simulation, $\beta$ is not sufficiently slowly varying relative to the solitary wave. Other simulations not reported here confirm this explanation as $T_{1}$ is varied. Note also that behind the solitary wave train, there is a rarefaction wave generated by the rear end of the initial "box-like" initial condition. Once the waves pass onto the shelf where $\beta=\beta_{1}$ is a constant, then the whole system will eventually transform into a finite number of rank-ordered solitary waves, as is well-known from the theory for a constant-coefficient $\mathrm{KdV}$ equation. This is already beginning to be apparent in the last panel of Fig. 1. The asymptotic description presented here is an intermediate phase where the undular bore is resolving incipient shock formation.

Importantly, although the leading solitary wave obeys the adiabatic law, the outcome for this solitary wave train is quite different from that for a single solitary wave. When a single solitary wave deforms adiabatically, it does so by conserving wave action, but cannot then also conserve mass. Instead, it generates a trailing shelf, which may itself then deform to generate a secondary solitary wave, and so on, see El and Grimshaw (2002), Grimshaw and Pudjaprasetya (2004). Here, mass is conserved by the whole wave train, as expressed in the Whitham modulation equations $(13,14,15)$ and their asymptotic reduction to $(18,20)$.

\subsection{Initial depression}

For the constant-coefficient $\mathrm{KdV}$ equation, when $U_{0}(\xi) \leq 0$,(depression) then no solitons are generated, and instead the solution disperses with the front being described by a nonlinear dispersing wave train, see Ablowitz and Segur (1977, 1981), Hammack and Segur (1978) for an analysis using the inverse scattering transform, or El and Khodorovsky (1993), El (2007) for an analysis based on the approach of Gurevich and Pitaevskii (1974) using the Whitham modulation theory. This has the shape of a leading rarefaction wave of depression, followed by a series of elevation waves riding on a negative pedestal.

Here, when the depth varies, we again adapt and simplify the Gurevich-Pitaevskii approach. Thus, as described above by (25), the solution initially develops according to the Hopf equation and a shock forms at a time $T_{0}$ and place $X_{0}(26)$. But in this depression case, the long-time evolution can be modelled as a leading rarefaction wave followed by a nonlinear wave train. The rarefaction wave solves the Hopf equation (24) which is the same as (18), and is also an exact solution of (5). It is given by $U=d$ where

$$
\begin{aligned}
& d=\frac{X-X_{0}}{6\left(T-T_{0}\right)}, \quad-H(T)<\frac{X-X_{0}}{6\left(T-T_{0}\right)}<0, \\
& \text { where } H=\left\{\frac{|M|}{3\left(T-T_{0}\right)}\right\}^{1 / 2}, \quad M=\int_{-\infty}^{\infty} U_{0}(X) \mathrm{d} X .
\end{aligned}
$$


Outside the domain $-H(T)<\left(X-X_{0}\right) /\left(6\left(T-T_{0}\right)\right)<0, d=0 . H(T)$ is determined by conservation of mass, where we assume that all the mass is carried by this rarefaction wave. Note that this part of the solution is independent of $\beta$. It is an $N$-wave, and at $X=-6 T H$, there is jump $H$ from the negative level $-H$ to 0 . The shock at the rear of the rarefaction wave has a speed of $\mathrm{d} X / \mathrm{d} T=-3 H$ and is resolved by an undular bore. The laboratory experiments of Hammack and Segur (1978) for a constant depth exhibit this behaviour, see their Figs. 2 and 3, also reproduced in the review by Arcas and Segur (2012), as also do the experiments by Klettner et al. (2012) for wave evolution on a slope. If $H$ was a constant, then the leading wave in the undular bore would be a solitary wave of amplitude $2 H$ relative to the pedestal of $-H$, see $\mathrm{El}$ (2007) for instance. However, although here $H$ depends on $T$ and decreases as $T^{-1 / 2}$ as $T$ increases, the theoretical results cited above confirm that is still essentially the case. A heuristic explanation of this is that since the total mass is carried by the rarefaction wave, the following wave train should overall have zero mass, implying that the waves should rise from $-H$ to $H$ and so on. The leading solitary wave has an amplitude of $-\left(X-X_{0}\right) / 3\left(T-T_{0}\right) \approx 2 H$ near the jump location, a speed $V=6 d+2 a=\left(X-X_{0}\right) / 3\left(T-T_{0}\right) \approx-2 H<0$ and hence begins to move up the rarefaction wave away from the jump position. But note that as $T \rightarrow \infty, H \rightarrow 0$ and so the solitary wave slows down and becomes smaller.

Next, to estimate the undular bore which follows, we use the solitary wave train system (14) and (21). When $d$ is given by (32), Eq. (21) becomes

$$
\mathcal{A}_{T}+\left(\frac{X-X_{0}}{T-T_{0}}+2 a\right) \mathcal{A}_{X}+\frac{\mathcal{A}}{T-T_{0}}=0,
$$

The general solution can be found using the transformation

$$
\mathcal{B}=\left(T-T_{0}\right) \mathcal{A}, \quad \xi=-\frac{X-X_{0}}{T-T_{0}}, \quad \rho=\int_{T}^{\infty} \frac{\mathrm{d} T^{\prime}}{\left(T^{\prime}-T_{0}\right)^{5 / 3} \beta^{1 / 3}\left(T^{\prime}\right)} .
$$

so that (33) becomes

$$
\mathcal{B}_{\rho}+2 \mathcal{B}^{2 / 3} \mathcal{B}_{\xi}=0
$$

This is a Hopf equation whose general solution can readily be found using characteristics. For our present purpose, it is sufficient note the long-time similarity solution

$$
\mathcal{B}=\left\{\frac{\xi}{2 \rho}\right\}^{3 / 2}, \quad \mathcal{A}=\frac{1}{T-T_{0}}\left\{\frac{\xi}{2 \rho}\right\}^{3 / 2}, \quad a=-\frac{1}{\left(T-T_{0}\right)^{5 / 3} \beta^{1 / 3}} \frac{X-X_{0}}{2 \rho} .
$$

It is useful to note here that

$$
\rho=\frac{3}{2 \beta^{1 / 3}\left(T-T_{0}\right)^{2 / 3}}+\int_{T}^{\infty} \frac{\beta_{T^{\prime}}\left(T^{\prime}\right) \mathrm{d} T^{\prime}}{2\left(T^{\prime}-T_{0}\right)^{2 / 3} \beta^{1 / 3}\left(T^{\prime}\right)} .
$$

In constant depth, or when $T>2 T_{1}, \beta$ is a constant, and then the second term in (37) is zero, so that $\rho=3 / 2 \beta^{1 / 3}\left(T-T_{0}\right)^{2 / 3}$ and the expression for the amplitude in (36) reduces to $a=-\left(X-X_{0}\right) / 3\left(T-T_{0}\right)$ in agreement with the asymptotic result from the exact theories cited above. Interestingly, this is independent of the constant value $\beta$, an outcome which is transparent for all solutions of (33), indicating that even when $\beta$ varies, the effect on the amplitude of the leading solitary wave is quite small. 
Fig. 2 Time series from the simulation with the initial condition (28) with $U_{M}=$

$-1, \Gamma_{0}=0.5, L=16$ and

$\beta_{1}=0.333, T_{1}=4, \kappa=0.75$.

$T=0, \beta=1, h=1$, top panel;

$T=T_{1}, \beta=-0.667, h=0.835$,

middle panel;

$T=2 T_{1}, \beta=0.333, h=0.614$, bottom panel
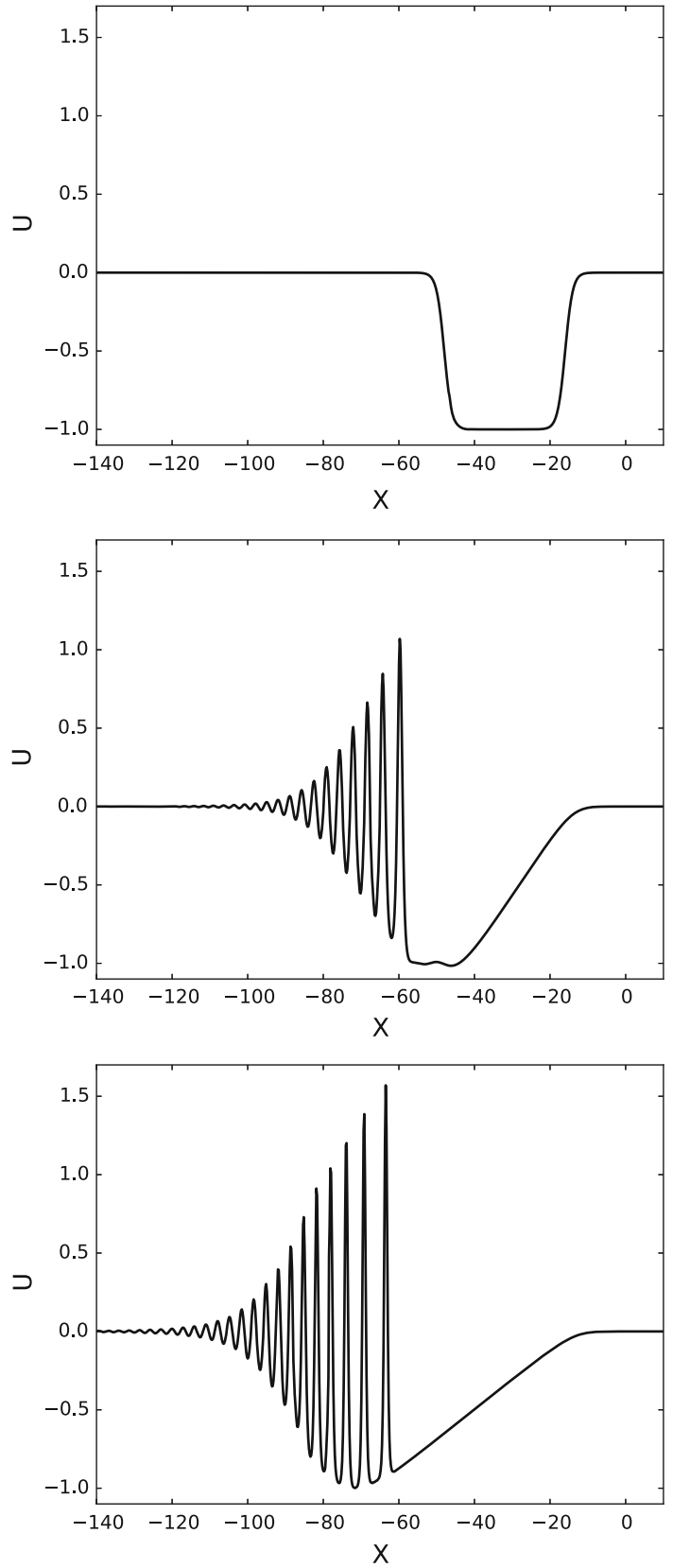

This asymptotic result holds only in the domain where (32) holds. The leading solitary wave is located at $\left(X-X_{0}\right) /\left(T-T_{0}\right) \approx-6 H(T)$, and the amplitude of this solitary relative to the pedestal is 
Fig. 3 Time series from the simulation with the initial condition (29) with $U_{M}=$

$1, \Gamma_{0}=0.5, L=16$ and

$\beta_{1}=0.333, T_{1}=4, \kappa=0.75$.

$T=0, \beta=1, h=1$, top panel;

$T=T_{1}, \beta=-0.667, h=0.835$,

middle panel;

$T=2 T_{1}, \beta=0.333, h=0.614$, bottom panel
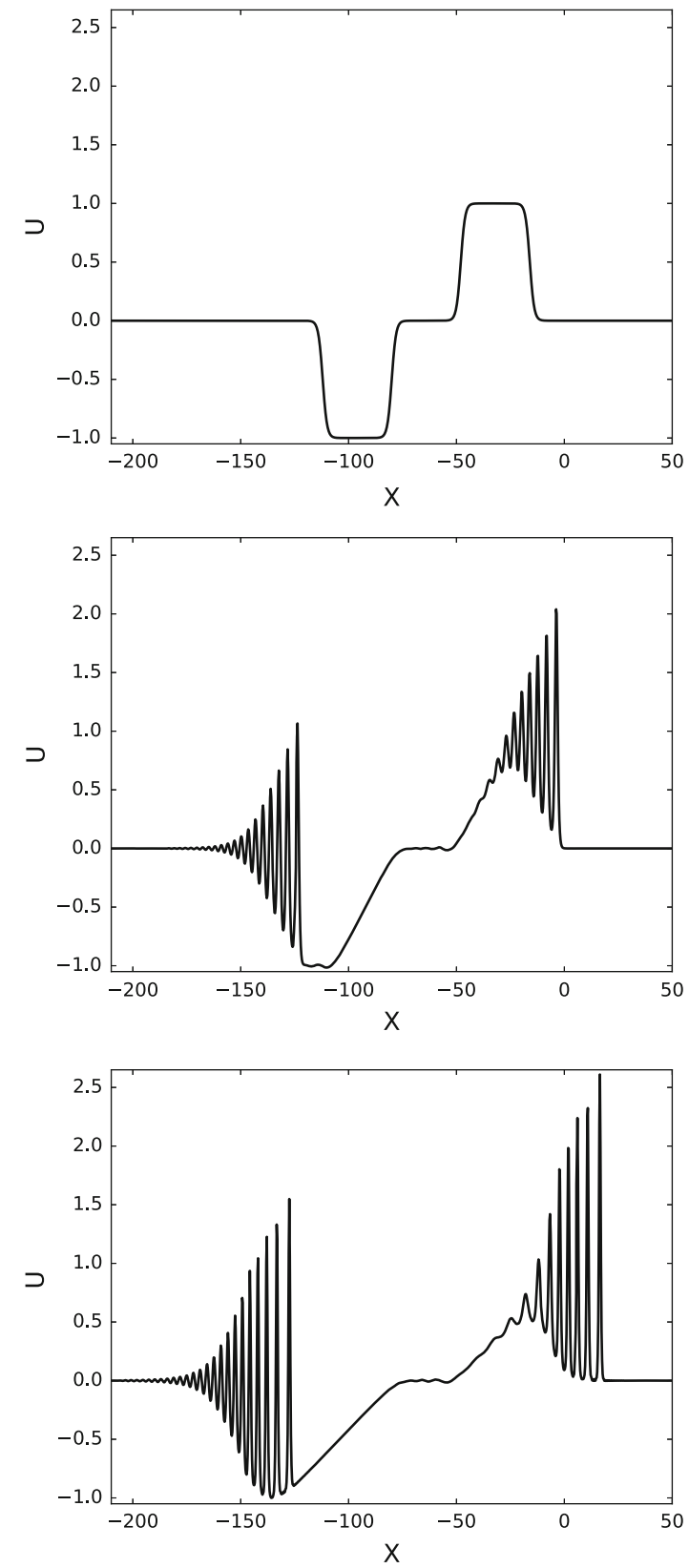

$$
a_{\max }=\frac{3 H(T)}{\beta^{1 / 3}\left(T-T_{0}\right)^{2 / 3} \rho} .
$$

When $\beta$ is a constant, this reduces to $2 H(T)$, and since this is also the case when $T \geq 2 T_{1}$, then 


$$
a_{\max }=\left\{\frac{4|M|}{3\left(T-T_{0}\right)}\right\}^{1 / 2}, \quad T \geq 2 T_{1} .
$$

We expect that for the remainder of the wave train, especially outside the domain where the rarefaction wave is defined, dispersion expressed through the value of $\beta$ will play a role. Thus, the amplitude of the leading solitary wave decays as $\left(T-T_{0}\right)^{-1 / 2}$ from a maximum value of $\left(16|M| / 27\left(2 T_{1}-T_{0}\right)\right)^{1 / 2}$ at $T=2 T_{1}$. This expression is formally independent of $\beta$, although $T_{1}$ does depend weakly on how the depth changes through expression (6) connecting $T$ with $x$. But we note that from (37) that since here $\beta_{T}<0,2 \beta^{1 / 3}\left(T-T_{0}\right)^{5 / 3} \rho<3\left(T-T_{0}\right)$ when $T<2 T_{1}$ indicating that (39) is an underestimate of the amplitude, which will in fact occur somewhere in the region $T<2 T_{1}$. Also, of course the shape of the leading solitary wave does vary with $\beta$ through the connection between the amplitude and wavenumber $k$ expressed in (19). Finally, we note that the general solution of (13) for the wavenumber is now

$$
k=\frac{\rho}{T-T_{0}} k_{0}\left(\frac{\left(X-X_{0}\right) \rho}{T-T_{0}}\right),
$$

where $k_{0}(\cdot)$ is again an arbitrary function. A typical simulation amongst several we have done is shown in Fig. 2 and is in very good qualitative agreement with this asymptotic theory. Quantitatively, the leading solitary wave has a predicted amplitude at $T=2 T_{1}$ of 2.41 (39), which is in good agreement with the value of approximately 2.3 in the simulation. Again, note that once the waves pass onto the shelf where $\beta=\beta_{1}$ is a constant, then the whole system will eventually transform into a nonlinear dispersing wave train according to the well-known theory for a constant-coefficient $\mathrm{KdV}$ equation. The asymptotic description presented here is an intermediate phase where the rarefaction wave and the following undular bore are resolving incipient shock formation.

\section{3 "Up-down" and “down-up" waves}

Finally, we show in Figs. 3 and 4 simulations for "up-down" and "down-up" waves, respectively. In the "up-down" wave case (Fig. 3), the initial elevation precedes the initial depression, and because the elevation component travels faster than the depression component, these two do not interact, and each evolves separately. Thus, the elevation and depression components in Fig. 3, respectively, completely agree with those in Figs. 1 and 2 for a single elevation and depression, respectively; note that the amplitude $U_{M}=1, L=16$ in the "up-down" case as in the separate elevation and depression wave cases. It follows that the asymptotic theories developed above in Sects. 4.1 and 4.2 can again be applied here to the separate elevation and depression components.

In the "down-up" wave case (Fig. 4), the amplitude $U_{M}=-1, L=16$ as in the separate depression and elevation wave cases. But now the initial depression precedes the initial elevation, and because the elevation component travels faster than the depression component, these two interact. The nature of the interaction can be inferred from the solution of the Hopf equation (24) for this case. This leading depression will develop into a depression rarefaction wave, followed by a shock, while the following elevation will develop into a shock, followed by an elevation rarefaction wave. In the absence of any dispersion, the shocks combine into a single stationary shock, of twice the magnitude of each component shock, preceded and followed by a rarefaction wave. Then, according to the Gurevich-Pitaevskii procedure, this shock will be resolved by an undular bore whose 
Fig. 4 Time series from the simulation with the initial condition (29) with $U_{M}=$

$-1, \Gamma_{0}=0.5, L=16$ and

$\beta_{1}=0.333, T_{1}=4, \kappa=0.75$.

$T=0, \beta=1, h=1$, top panel;

$T=T_{1}, \beta=-0.667, h=0.835$,

middle panel;

$T=2 T_{1}, \beta=0.333, h=0.614$, bottom panel
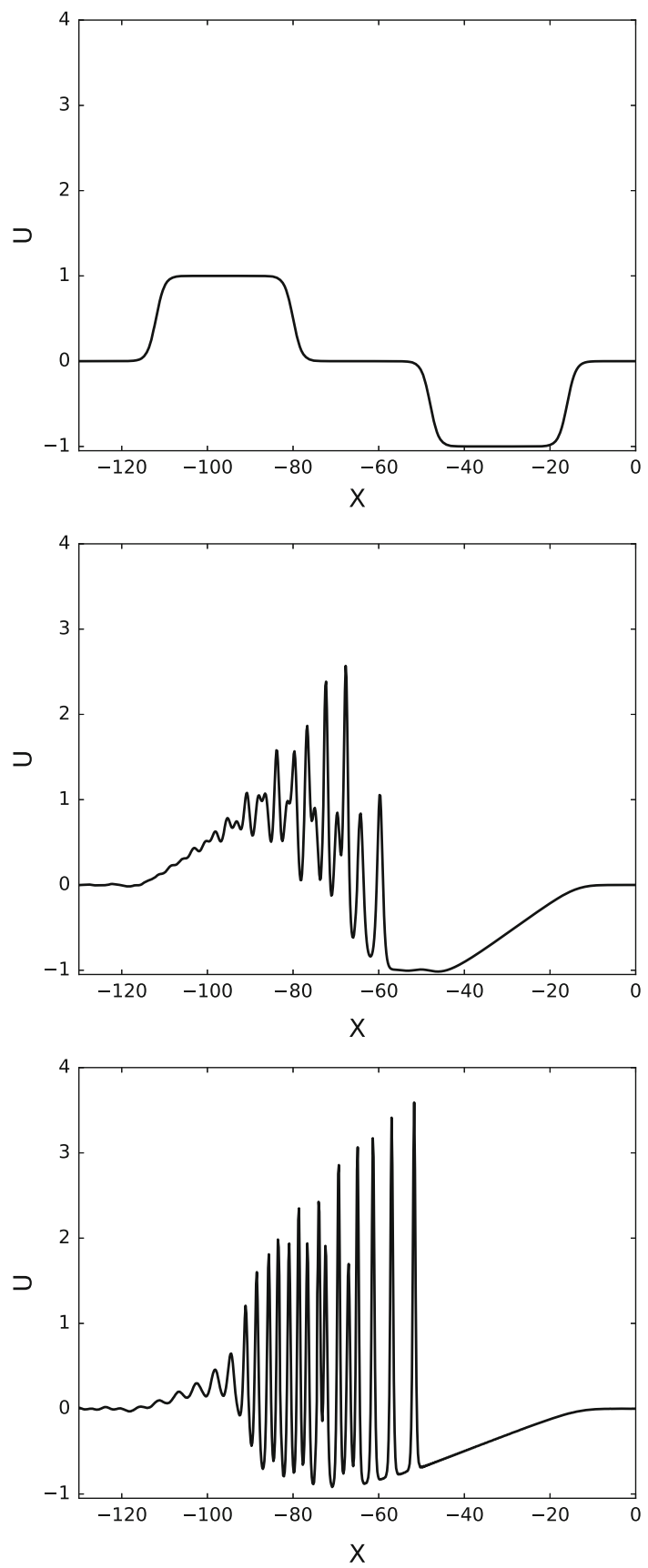

leading solitary wave will have an amplitude twice that of the shock. The outcome is thus a solitary wave whose amplitude may be twice that from either an elevation or depression initial condition alone. The simulations shown in Fig. 4 show some qualitative evidence of this process. At the intermediate time $T=T_{1}$, we can see the leading and trailing 
rarefaction waves but the solitary wave trains from each component are already interacting. At the final time $T=2 T_{1}$, the leading and trailing rarefaction waves are still visible, and the interacting solitary wave trains have begun to merge into a nonlinear wave train with three parts. The leading part is a single solitary wave train, with evidence that the leading wave has amplitude of approximately 4, which is four times the initial amplitude. The trailing part shows a structure similar to that for the trailing part of an undular bore. The middle part is a rather complex interaction between the other two parts.

\section{Discussion}

In this work, we have examined how tsunami waves deform as they propagate shorewards over the continental slope and shelf, using the framework of the vKdV equation (1). For analytical and numerical convenience, we have used the transformed equation (5). We have constructed asymptotic solutions describing the evolution of a tsunami wave over a slope onto a shelf, using the Gurevich-Pitaevskii method based on the Whitham modulation theory, reduced here to the solitary wave train equations $(13,18,21)$ which are adequate for the description of the leading waves. This is a very useful reduction; as unlike the full Whitham modulation equations, this reduced set can be solved as a sequence of Hopf equations for the mean level $d$, then the wave amplitude $a$ and then finally the wavenumber $k$. We have used this procedure for the case when the initial wave is one of the elevation in Sect. 4.1, an initial depression in Sect. 4.2 and initial "up-down" and "down-up" waves in Sect. 4.3. In each case, the asymptotic analysis is compared favourably with our numerical simulations.

Although we have found it convenient in the theoretical development and in the numerical simulations to use the canonical $\mathrm{KdV}$ equation (5), in presenting a summary of the obtained results we use the original physical variables, expressed through the transformations $(3,4,6)$. Also, as discussed above, the plots of our numerical simulations are time series at three fixed places, namely at the base of the slope, around the middle of the slope and at the end of the slope where the depth becomes constant. Our main findings relevant for tsunamis are:

- The amplitude of the leading wave emerging from an initial elevation is determined by the maximum initial amplitude $\zeta_{M}$ and increases as the depth decreases, $\zeta \sim 2 \zeta_{M} / h$. The mass $M>0$ of the initial elevation determines the number of waves in the following wave train.

- The amplitude of the leading wave emerging from an initial depression is determined by the total initial mass $M<0, \zeta \sim(3|M| / T)^{1 / 2} h^{-1 / 4}$, where $T$ is defined by (6).

- For a tsunami approaching the shore with a rather small amplitude $\zeta_{M}$ but quite long wavelength, so quite large mass $M$, these imply that a depression wave may be more destructive than an elevation wave.

- An initial "down-up" wave can produce a leading wave twice as large as that from a single elevation or depression, and with a fourfold increase in amplitude.

In order to express our results in quantitative terms, we note that if $U(X, T)$ is a solution of $\mathrm{KdV}$ equation (5) then so is $\epsilon^{2} U\left(\epsilon X, \epsilon^{3} T\right)$ provided that $\beta(T)$ is replaced by $\beta\left(\epsilon^{3} T\right)$. Here $\epsilon \ll 1$ is a free parameter measuring the wave nonlinearity and dispersion and is implicitly present in the derivation of the suite of $\mathrm{KdV}$ equations presented here. For example, choose $\epsilon=0.1$, then the simulations for an initial elevation or depression wave shown in Figs. 1 
and 2 correspond to an initial wave of height $\pm 1 \mathrm{~m}$ on a depth $h_{0}=100 \mathrm{~m}$ say, and an initial wavelength of $160 \mathrm{~m}$. For the simulations, $\beta_{1}=1 / 3$, which corresponds to $h_{1}=0,61, h_{0}=61 \mathrm{~m}$. From the simulations, the elevation wave then grows to a leading solitary wave amplitude of $1.9 \mathrm{~m}$, while the depression wave grows to $1.7 \mathrm{~m}$ at the location where the depth is $61 \mathrm{~m}$. But if instead the initial wavelength is $640 \mathrm{~m}$, then the initial mass is doubled, and the depression wave will grow to $3.4 \mathrm{~m}$. Also the dimensional length of the slope is $x_{s} h_{0} \epsilon^{-1}=34.5 \mathrm{~km}$. This indicates inter alia that the nonlinear and dispersive effects contained in the $\mathrm{KdV}$ model can indeed develop over plausible distances when applied as here to the near shore region. In much deeper water, where say $h_{0}=$ $1.6 \mathrm{~km}, \epsilon=0.02$ for an initial amplitude of again $1 \mathrm{~m}$, the corresponding length scale $x_{s} h_{0} \epsilon^{-1}=2760 \mathrm{~km}, 80$ times larger, indicating inapplicability of a KdV model, as argued in a different manner by Madsen et al. (2008), Madsen and Schaffer (2010) and Arcas and Segur (2012) for instance.

As deep ocean tsunamis which impact the continental slope and shelf are typically of quite small amplitude, that is, $\left|\zeta_{M}\right| \ll h_{0}$, but have very long wavelengths so that the mass $|M|$ is large, the results obtained here suggest that depression waves may be more destructive than elevation waves, and that the combination of a "down-up" wave is even more destructive. But we caution that in this KdV framework, for the emerging waves from an initial depression, even if the amplitudes become quite large at some intermediate time, the whole wave train will eventually disperse. On the other hand, the emerging waves from an initial elevation are solitary waves, which grow as $h^{-1}$ and maintain their shape. Finally, we note that in a higher-order KdV model Grimshaw et al. (2015) found "down-up" waves with a fourfold amplification, but more persistent than those we have examined here.

Acknowledgments R.G. was supported by the Leverhulme Trust through the award of a Leverhulme Emeritus Fellowship. C.Y. is supported by the CSC (Chinese Scholarship Council).

Open Access This article is distributed under the terms of the Creative Commons Attribution 4.0 International License (http://creativecommons.org/licenses/by/4.0/), which permits unrestricted use, distribution, and reproduction in any medium, provided you give appropriate credit to the original author(s) and the source, provide a link to the Creative Commons license, and indicate if changes were made.

\section{References}

Ablowitz MJ, Segur H (1977) Asymptotic solutions of the Korteweg-de Vries equation. Stud Appl Math 57:13-44

Ablowitz MJ, Segur H (1981) Solitons and the inverse scattering transform. SIAM, Philadelphia

Arcas D, Segur H (2012) Seismically generated tsunamis. Philos Trans R Soc 370:1505-1542

Carrier G, Wu T, Yeh H (2003) Tsunami run-up and drawdown on a plane beach. J Fluid Mech 475:79-99

Charvet I, Eames I, Rossetto T (2013) New tsunami run-up relationships based on long wave experiments. Ocean Model 69:79-92

Dias F, Dutykh D, O’Brien L, Renzi E, Stefanakis T (2014) On the modelling of tsunami generation and tsunami inundation. Procedia IUTAM 10:338-355

Didenkulova I (2009) New trends in the analytical theory of long sea wave runup. In: Quak E, Soomere T (eds) Applied wave mathematics: selected topics in solids, fluids, and mathematical methods. Springer, Berlin, pp 265-296

Didenkulova I, Pelinovsky E (2011) Nonlinear wave evolution and run-up in an inclined channel of a parabolic cross-section. Phys Fluids 23:086602

Didenkulova I, Zahibo N, Kurkin A, Levin B, Pelinovsky E, Soomere T (2006) Runup of nonlinearly deformed waves on a coast. Doklady Earth Sci 411:1241-1243

Didenkulova I, Pelinovsky E, Soomere T, Zahibo N (2007) Runup of nonlinear asymmetric waves on a plane beach. In: Kundu A (ed) Tsunami and nonlinear waves. Springer, Berlin, pp 175-190 
Didenkulova I, Pelinovsky E, Didenkulov O (2014) Run-up of long solitary waves of different polarities on a plane beach. Izvest Atmos Ocean Phys 50:532-538

Drazin PG, Johnson RS (1989) Solitons: an introduction. CUP, Cambridge

El G (2007) Kortweg-de Vries equation and undular bores. In: Grimshaw R (ed) Solitary waves in fluids. Advances in fluid mechanics, vol 47. WIT Press, Ashurst, pp 19-53

El GA, Grimshaw R (2002) Generation of undular bores in the shelves of slowly-varying solitary waves. Chaos 12:1015-1026

El GA, Khodorovsky VV (1993) Evolution of a solitonless large-scale perturbation in Korteweg-de Vries hydrodynamics. Phys Lett A 182:49-53

El G, Grimshaw RHJ, Kamchatnov AM (2007) Evolution of solitary waves and undular bores in shallowwater flows over a gradual slope with bottom friction. J Fluid Mech 585:213-244

El G, Grimshaw R, Tiong W (2012) Transformation of a shoaling undular bore. J Fluid Mech 709:371-395

Fernando H, Braun A, Galappatti R, Ruwanpura J, Wirisinghe SC (2008) Tsunamis: manifestation and aftermath. In: el Hak MG (ed) Large scale disasters. Cambridge University Press, Cambridge, pp 258-292

Grimshaw R (1979) Slowly varying solitary waves i. Korteweg-de Vries equation. Proc R Soc 368A:359-375

Grimshaw R (1981) Evolution equations for long nonlinear internal waves in stratified shear flows. Stud Appl Math 65:159-188

Grimshaw R (2007a) Internal solitary waves in a variable medium. Gesellschaft fur Angewandte Mathematik 30:96-109

Grimshaw R (2007b) Solitary waves propagating over variable topography. In: Kundu A (ed) Tsunami and nonlinear waves. Springer, Berlin, pp 49-62

Grimshaw RHJ, Pudjaprasetya SR (2004) Generation of secondary solitary waves in the variable-coefficient Korteweg-de Vries equation. Stud Appl Math 112:271-279

Grimshaw R, Pelinovsky E, Talipova T, Kurkina A (2010) Internal solitary waves: propagation, deformation and disintegration. Nonlinear Process Geophys 17:633-649

Grimshaw RHJ, Hunt JCR, Chow KW (2015) Changing forms and sudden smooth transitions of tsunami waves. J Ocean Eng Mar Energy 1:145-156

Grue J, Pelinovsky E, Fructus D, Talipova T, Kharif C (2008) Formation of undular bores and solitary waves in the Strait of Malacca caused by the 26 December 2004 Indian Ocean tsunami. J Geophys Res 113:C05008

Gurevich AV, Pitaevskii LP (1974) Nonstationary structure of a collisionless shock wave. Sov Phys JETP 38:291-297

Hammack JL, Segur H (1978) The Korteweg-de Vries equation and water waves. III. Oscillatory waves. J Fluid Mech 84:337-358

Johnson RS (1973a) On an asymptotic solution of the Korteweg-de Vries equation with slowly varying coefficients. J Fluid Mech 60(4):813-82414

Johnson RS (1973b) On the development of a solitary wave moving over an uneven bottom. Proc Camb Philos Soc 73:183-203

Kamchatnov AM (2000) Nonlinear periodic waves and their modulations. An introductory course. World Scientific, Singapore

Kamchatnov AM (2004) On Whitham theory for perturbed integrable equations. Phys D 188:247-261

Klettner C, Balasubramanian S, Hunt J, Fernando H, Voropayaev S, Eames I (2012) Draw-down and run-up of tsunami waves on sloping beaches. Eng Comp Mech 165:119-129

Kobayashi N, Lawrence AR (2004) Cross-shore sediment transport under breaking solitary waves. J Geophys Res 109:C03047

Kundu A (2007) Tsunamis and nonlinear waves. Springer, Berlin

Madsen P, Schaffer H (2010) Analytical solutions for tsunami run-up on a plane beach: single waves, $\mathrm{N}$-waves and transient waves. J Fluid Mech 645:27-57

Madsen PA, Fuhrman DR, Schaffer HA (2008) On the solitary wave paradigm for tsunamis. J Geophys Res 113:C12012

Myint S, Grimshaw R (1995) The modulation of nonlinear periodic wavetrains by dissipative terms in the Korteweg-de Vries equation. Wave Motion 22:215-238

Pelinovsky E (2006) Hydrodynamics of tsunami waves. In: Grue J, Trulsen K (eds) Waves in geophysical fluids: CISM courses and lectures, No. 489. Springer, Berlin, pp 1-48

Rossetto T, Allsop W, Charvet I, Robinson DI (2011) Physical modelling of tsunami using a new pneumatic wave generator. Coast Eng 58:517-527

Segur H (2007) Waves in shallow water, with emphasis on the tsunami of 2004. In: Kundu A (ed) Tsunami and nonlinear waves. Springer, Berlin, pp 3-29 
Soloviev S, Mazova R (1994) On the influence of sign of leading tsunami wave on run-up height on the coast. Sci Tsunami Hazards 12:25-31

Tadepalli S, Synolakis C (1994) The run-up of N-waves on sloping beaches. Proc R Soc A 445:99-112

Tadepalli S, Synolakis C (1996) Model for the leading wave of tsunamis. Phys Rev Lett 77:2141-2144

Whitham GB (1965) Non-linear dispersive waves. Proc R Soc Lond A 283:238-261

Whitham GB (1974) Linear and nonlinear waves. Wiley, New York 